\title{
PSORÍASE PUSTULOSA PALMOPLANTAR NA INFÂNCIA: UM RELATO DE CASO
}

\section{PALMOPLANTAR PUSTULAR PSORIASIS IN CHILDHOOD: A CASE REPORT}

\author{
Aline Rodrigues Dantas ${ }^{1}$, Wollia Monik Vaz Fernandes ${ }^{1}$, Edilberto \\ Vasconcelos Pereira Júnior ${ }^{1}$, Nicole Rangely Nogueira Martins De \\ Carvalho ${ }^{2}$.
}

\begin{abstract}
RESUMO
Introdução: A psoríase pustulosa palmoplantar, também conhecida como pustulose palmoplantar (PPP) é uma forma de dermatose psoriasiforme caracterizada por erupções de pústulas estéreis de localização palmoplantar e simétrica. Sua prevalência é estimada entre 0,01 a 0,05\%, afetando principalmente adultos do sexo feminino entre a sexta e a sétima décadas de vida. A doença é rara na faixa etária pediátrica. Desenvolvimento: No presente trabalho foi relatado um raro caso de PPP, em criança de 6 anos, destacando seu manejo hospitalar e atentando para sua dificuldade diagnóstica. Conclusão: o diagnóstico de PPP ainda é de difícil realização no Brasil. Isso pode ter relação com o perfil epidemiológico territorial, que levaria primeiramente a diagnósticos de etiologia infecciosa. Consideramos que seja pertinente um aumento do número de estudos sobre a PPP, o que ajudaria no diagnóstico precoce e redução das dificuldades físicas e psicossociais que o paciente enfrenta devido à doença.

Palavras-chave: psoríase; criança; dermatopatia.
\end{abstract}

\section{ABSTRACT}

Introduction: The palmoplantar pustular psoriasis, also known as palmoplantar pustulosis (PPP), is a psoriasiform dermatosis characterized by eruptions of sterile pustules with a symmetrical and palmoplantar location. It's prevalence is estimated between 0,01 to $0,05 \%$, mainly affecting female adults in the sixth and seventh decades of life. The disease is rare in the pediatric age. Development: In the present work it was reported a rare case of PPP, in a 6 years old child, highlighting its hospital management and paying attention to the difficulty in diagnosis. Conclusion: the diagnosis of PPP is still difficult to achieve in Brazil. This may have correlation with the territorial epidemiological profile, which would lead primarily to diagnostics of infectious etiology. We consider to be relevant an increase in the number of studies about PPP, which would help with early diagnosis and reduction of the physical and psychosocial difficulties that the patient faces due to the disease.

Keywords: psoriasis; child; skin diseases.
Autor correspondente: Aline Rodrigues Dantas; ard.aline@gmail.com

Editor: Guedes V. R. Medicina, Universidade Federal do Tocantins, Brasil.

Publicado: 26 de setembro de 2017.

Direitos Autorais: (c) 2017 Dantas et al. Este é um artigo de acesso aberto que permite o uso, a distribuição e a reprodução sem restrições em qualquer meio, desde que o autor original e a fonte sejam creditados.

Conflito de interesses: os autores declararam que não existem conflitos de interesses. 


\section{INTRODUÇÃO}

A psoríase é uma doença imunoinflamatória crônica da pele e das articulações, com grande polimorfismo de expressão clínica, caracterizada pela hiperplasia epidérmica ${ }^{1,2}$. De ocorrência universal e prevalência nos EUA e Europa entre 2 a $4 \%^{3}$, estima-se que afeta cerca de $1 \%$ da população brasileira ${ }^{1}$. Na população pediátrica a prevalência estimada varia de $0,55 \%$ a $1,4 \%^{4}$.

A psoríase pustulosa palmoplantar, também conhecida como pustulose palmoplantar (PPP), é uma forma de dermatose psoriasiforme originalmente classificada no espectro da psoríase, ainda que alguns autores a considerem uma entidade distinta a partir de uma proposta do International Psoriasis Council (Conselho Internacional de Psoríase), sendo sua classificação ainda campo de intenso debate $^{5,6}$

A PPP é caracterizada por erupções de pústulas estéreis de localização palmoplantar e simétrica. Tem prevalência estimada entre 0,01 a $0,05 \%{ }^{6}$ e afeta principalmente adultos do sexo feminino entre a sexta e sétima décadas de vida ${ }^{7}$. Possui rara incidência na faixa etária pediátrica ${ }^{8}$. As lesões geralmente são bastante dolorosas e diminuem de forma substancial a qualidade de vida ${ }^{5,6}$. Há ainda, a possibilidade de coexistência de lesões de psoríase vulgar e outras semelhantes ao eczema em distintas partes do corpo ${ }^{7}$.

A doença geralmente tem um curso crônico e recidivante e é resistente ao tratamento ${ }^{7}$. Como principais fatores precipitantes são relatados o tabagismo; a presença de infecções, mais comumente tonsilite; a sinusite e as infecções dentárias $^{9}$; a sensibilidade a metais como níquel, cromo e cobalto; o fenômeno de Koebner, desencadeado pela estimulação mecânica, mais comumente em região plantar por sapatos $^{5}$; as condições de estresse psicológico, como ansiedade e distúrbios psicossomáticos ${ }^{8}$; e além disso, tem-se também a influência sazonal ${ }^{5}$.

Diante do exposto, observa-se a raridade da doença apresentada, principalmente quando seu acometimento é observado ainda na infância. O objetivo do presente relato de caso é descrever e discutir o quadro de apresentação da doença de nossa paciente, uma criança de 6 anos, diante de uma breve revisão da literatura disponível sobre PPP e destacar ao longo do texto a dificuldade diagnóstica da patologia e o tratamento empregado no caso.

\section{RELATO DO CASO}

Criança do sexo feminino, 6 anos de idade, acompanhada pela mãe, foi atendida no Hospital Infantil Público de Palmas - TO, queixando-se de aparecimento, há 7 dias da internação, de lesões pustulosas sobre base eritematodescamativa difusamente em regiões plantares e laterais de ambos os pés, com evolução rápida para crostas, associadas a prurido, dor intensa e secreção purulenta local de odor fétido (Figura 1). Negou febre e lesões em outros locais. Relatou que fez uso de Azitromicina oral neste período devido otite média direita. Apresentava ainda cáries dentárias, pediculose e sinais de subnutrição. Negou tabagismo ativo ou passivo.

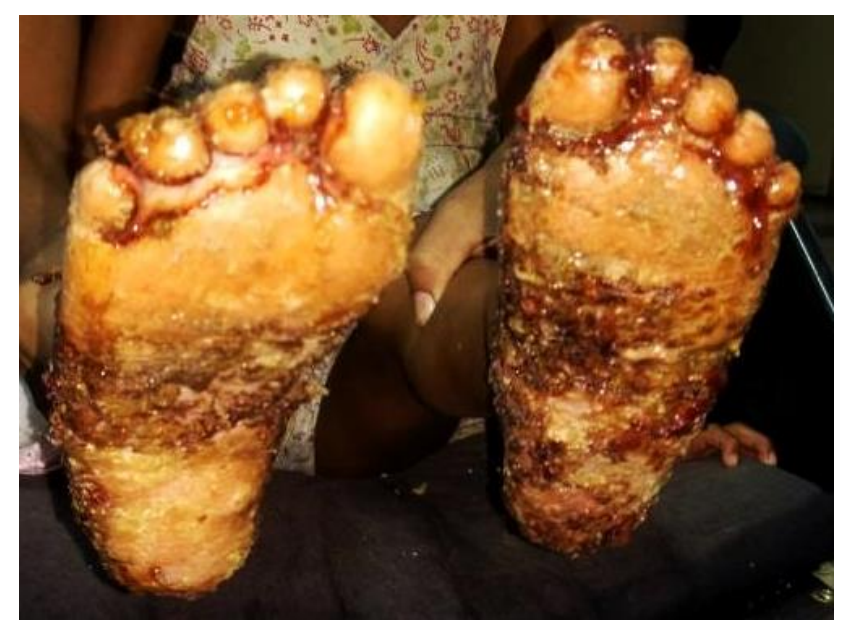

Figura 1. Aspecto das lesões no dia da chegada ao hospital.

A mãe da paciente relatou que a filha apresenta quadros similares recorrentes, desde 1 ano e 6 meses de idade, tendo ocorrido o último episódio há 2 meses. Durante as crises, procurava o serviço de assistência básica de saúde onde na maioria das vezes era tratada para micose e/ou hipovitaminose, sendo que o quadro levemente regredia, porém em seguida retornava. Mãe informou que dois irmãos da paciente também apresentam sintomatologia semelhante.

Dentre os exames laboratoriais relevantes da paciente foi encontrada leucocitose de $18800 / \mathrm{mm}^{3}$ (VR: 5000 a $\left.11000 / \mathrm{mm}^{3}\right)$, às custas de segmentados $\left(11468 / \mathrm{mm}^{3}-\right.$ VR:

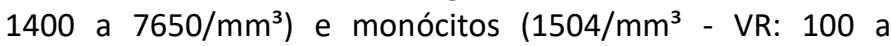
$1360 / \mathrm{mm}^{3}$ ) e proteína $C$ - reativa (PCR) aumentada de 47,61 $\mathrm{mg} / \mathrm{l}(\mathrm{VR}:<6 \mathrm{mg} / \mathrm{l})$, sem outros comemorativos.

Internada no referido hospital, a criança foi avaliada pelas especialidades de Dermatologia e Pediatria, que aventaram a hipótese de psoríase pustulosa palmoplantar infectada em regiões plantares. Foram inicialmente prescritos banhos de imersão dos pés em água morna com permanganato de potássio 1:4 litros por quatro vezes ao dia, uso tópico de Neomicina e Colagenase duas vezes ao dia, além de limpeza local e curativo oclusivo nos pés, com gazes estéreis. Iniciou-se também antibioticoterapia endovenosa (que ao final do tratamento totalizou 7 dias) com Cefalotina de 6 em 6 horas e Clindamicina de 8 em 8 horas. Cerca de 38 horas depois da internação, foram realizados desbridamento das lesões em centro cirúrgico e biópsia de material para exame histopatológico. Após, os banhos de imersão passaram a ser feitos sem permanganato de potássio e em seguida eram aplicadas camada de Colagenase e gazes com Vaselina, sendo feita devida oclusão com faixas, duas vezes ao dia.

Paciente evoluiu bem, sem intercorrências, com significativa melhora do aspecto da pele plantar (Figura 2), ainda sensível e com descamação, mas em boa recuperação e grande melhora da sintomatologia. Recebeu alta hospitalar após nove dias de internação, com orientações para manter em domicílio limpeza, curativo com Colagenase e uso de meias grossas durante todo o dia, até melhora completa do quadro, além de ser encaminhada para acompanhamento ambulatorial com dermatologista. 


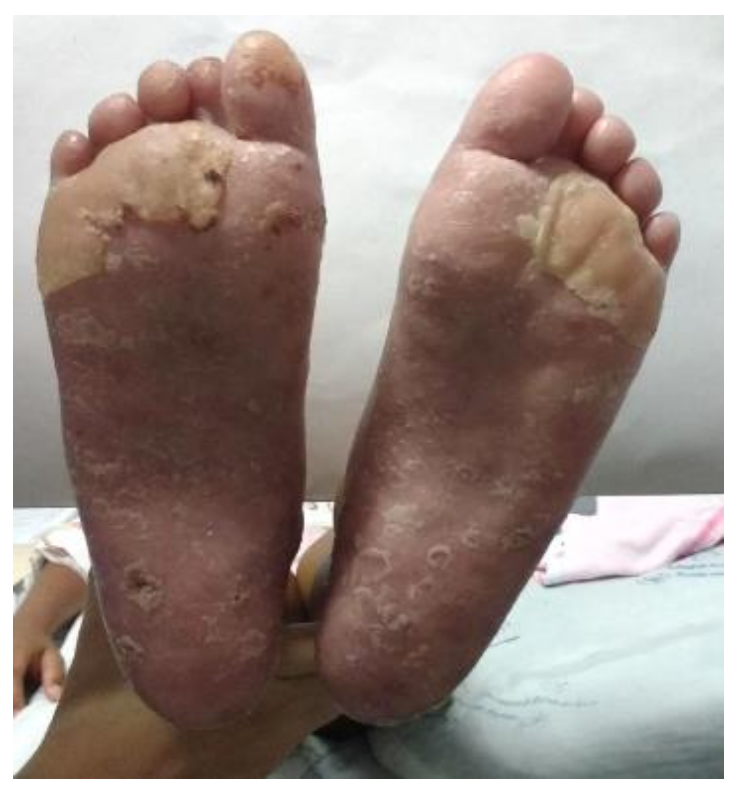

Figura 2. Regiões plantares no dia da alta hospitalar, com melhora do aspecto lesional.

O resultado (obtido após a alta hospitalar) do exame histopatológico da pele de região plantar evidenciou camada córnea hiperceratótica e áreas de paracetose, com epiderme apresentando hiperplasia psoriasiforme com focos de exocitose de neutrófilos e linfócitos. $\mathrm{Na}$ derme papilar, teleangiectasia e infiltrado inflamatório superficial linfocitário leve perivascular e difuso. Este resultado foi compatível com a suspeita clínica de pustulose palmoplantar.

\section{DISCUSSÃO}

A pustulose palmoplantar é caracterizada por uma erupção crônica de pústulas estéreis nas palmas das mãos e plantas dos pés, observadas em um fundo de descamação e eritema, que podem se espalhar para a região lateral dessas extremidades ${ }^{7}$.

O diagnóstico diferencial de pustulose palmoplantar inclui infecção fúngica superficial, erupção bacteriana, disidrose $\mathrm{e}^{10}$, acrodermatite contínua de Hallopeau, psoríase anular e dermatite de contato irritante ${ }^{1,2,11,7}$.

A doença geralmente cursa com resistência ao tratamento e remissões frequentes ${ }^{7}$, sendo essas observadas na maioria dos pacientes por curtos períodos, de no máximo um ano, no qual as lesões geralmente coalescem e se resolvem, resultando em uma descoloração amarronzada ${ }^{6}$. Na história da paciente somente no início de 2017 foram três episódios em quatro meses. 0 curso da doença não foi afetado pelas diversas tentativas de tratamento - antifúngicos, antibióticos e hidratantes contendo uréia - empregadas antes do diagnóstico de PPP.

Na PPP as pústulas na região palmar e plantar podem ter aparência normal ou inflamada e apresentar fissuras dolorosas, prurido e sensação de queimação, sendo a queixa principal de dor. Isso ajuda a diferenciar de infecções fúngicas, dermatite de contato e psoríase palmoplantar que se apresentam principalmente com prurido ${ }^{7}$. A nossa paciente apresentava PPP infectada, provavelmente devido a diagnóstico e orientação inadequados, que propiciaram a exposição prolongada de fissuras e pústulas abertas à microorganismos diversos.

Apesar da relativamente pequena área de superfície corporal afetada, os pacientes com PPP comumente se deparam com dificuldade para andar e redução de destreza manual, além de lidar com o estigma e os aspectos psicossociais da doença, ${ }^{5,9}$. Portanto, nossa paciente permaneceu em quarto privativo durante o início do tratamento hospitalar, objetivando proteção social da mesma.

No estudo realizado por Kubota et al. de $2015^{3}$, que utilizou a base de dados Nacional do Japão para estimar a prevalência Nacional de psoríase e PPP no país, foi encontrada prevalência de $0,12 \%$ para PPP, sendo que desses apenas $1,75 \%$ tinham idade entre 0 a 19 anos, indicando a raridade da doença em crianças. $O$ estudo revelou ainda sazonalidade nas exacerbações, também observada na paciente descrita pelo presente relato, em que a doença parece ter períodos de exacerbações mais frequentes em épocas mais quentes, em concordância com estudos que referem que a doença é mais comum em localizações com clima quente e úmido ${ }^{5,6}$.

Também sabe-se que a psoríase apresenta base hereditária, provavelmente multifatorial, o que significa dizer que é de herança poligênica e requer fatores ambientais para sua expressão ${ }^{2}$.

Infecções localizadas são bem caracterizadas como fator desencadeante por diversos estudos ${ }^{6,7,9}$. Nossa paciente apresentava concomitantemente à PPP, cárie dentária, otite média e pediculose. Embora existam estudos que relacionem PPP à sinusite ${ }^{9}$ não se pode estender os achados à otite média, mesmo essa tendo sua etiopatogenia interligada à sinusite e ainda são necessários estudos para indagar o papel das parasitoses cutâneas no desencadeamento da PPP.

Conquanto a fisiopatologia da psoríase pustulosa palmoplantar permaneça obscura, é amplamente aceito que a glândula sudorípara écrina é significativa para a sua patogênese. $O$ acrossiríngeo (a porção mais intraepidérmica da glândula sudorípara écrina) serve como o principal local de inflamação e formação de pústulas ${ }^{5}$. Um antígeno desconhecido pode ativar glândulas sudoríparas e dutos causando inflamação no acrossiríngeo e o desenvolvimento de lesões cutâneas. Níveis elevados de interleucinas (IL) IL-8 e IL17 atrairiam neutrófilos levando a formação de pústulas. Além das infecções, fumar pode alterar a ação de neutrófilos em glândulas écrinas, participando do processo inflamatório na pele e na etiopatogenia da PPP ${ }^{7}$.

Existem vários subtipos clínicos da psoríase, incluindo as variantes pustulares incomuns, que são subdivididas em generalizadas e formas localizadas. As formas localizadas incluem a psoríase pustulosa palmoplantar e a Acrodermatite de Hallopeau ${ }^{12}$ - quando a erupção das pústulas acontece no leito ungueal (gerando distrofia) e nas extremidades dos dedos. Os subtipos variam em suas apresentações, mas todos possuem características histopatológicas similares ${ }^{5}$.

O diagnóstico da PPP é feito pelo exame físico e análise anatomopatológica evidenciando paraqueratose, e espongiose epidérmica com acúmulo de neutrófilos na epiderme ${ }^{10}$. 0 exame histológico demonstra pústulas intraepidérmicas, com polimorfonucleares na periferia, além de alterações espongiformes. Há evidências de mastócitos e eosinófilos 
abaixo da pústula, entretanto, no interior da mesma 2a ed. Rio de Janeiro: Sociedade Brasileira de Dermatologia; encontram-se neutrófilos e eosinófilos ${ }^{6}$. 2012. 172 p.

Devido à patogênese da doença, o objetivo da 2. Azulay RD, Azulay DR, Azulay LA-A, editors. Dermatologia. 6á terapêutica é o seu controle clínico, de preferência com longo ed. Rio de Janeiro: Guanabara Koogan; 2013.1156 p.

período de remissão e reflexos positivos na qualidade de vida ${ }^{2}$. 3. Kubota K, Kamijima Y, Sato T, Ooba N, Koide D, lizuka H, Incluindo a ênfase na educação familiar para lidar com os Nakagawa H.Epidemiology of psoriasis and palmoplantar aspectos emocionais da doença, caráter crônico e a tendência à remissão espontânea, além da eliminação de fatores desencadeantes e agravantes ${ }^{8}$.

A PPP é reconhecida como de difícil controle e tratamento. Nenhuma diretriz terapêutica foi ainda definida, praticamente todas as opções de tratamento conhecidas de psoríase ou outras doenças autoimunes têm sido tentadas para a $\mathrm{PPP}^{13}$

A região palmoplantar por apresentar o estrato córneo mais espesso, representa uma barreira para ação adequada de agentes no tratamento tópico, fato que explica a eficácia moderada destas formas de tratamento. Tratamentos sistêmicos para a PPP têm mostrado eficácia limitada quando comparados a outras lesões psoriasiformes ${ }^{11}$.

Em adultos, o tratamento inclui fototerapia, fotoquimioterapia e agentes sistêmicos como retinóides, metotrexato, ciclosporina e agentes biológicos, como alefacept, infliximab, adalimumab e efalizumab ${ }^{5,10,14}$. Contudo, estudos sobre o uso prolongado, toxicidade sistêmica e experiência clínica em crianças são limitados e escassos, o que dificulta o uso seguro dessas opções terapêuticas nessa faixa etária ${ }^{8}$.

$\mathrm{Na}$ infância, o tratamento da PPP tende a ser conservador, com uso limitado de corticóides tópicos, agentes emolientes e cuidados paliativos ${ }^{8}$, podendo se estender para fototerapia, uso de retinóides, ciclosporina, metotrexato e agentes biológicos em casos de difícil controle ${ }^{13}$.

O tratamento de nossa paciente após a abordagem intra hospitalar já descrita, seria a continuidade do cuidado com agentes emolientes e proteção local e segmento com dermatologista, que nos informou haver a possibilidade de início do tratamento com Metotrexato.

\section{CONCLUSÃO}

Diante do exposto pelo atual relato de caso, podemos inferir que o diagnóstico da PPP ainda é de difícil realização no Brasil, principalmente quando realizado por profissionais não especializados. Isso pode ter relação com a baixa prevalência da doença, tanto na faixa etária da paciente aqui descrita quanto no perfil epidemiológico territorial, que levariam primeiramente a diagnósticos de etiologia infecciosa.

Consideramos pertinente o aumento do número de estudos sobre a Pustulose Palmoplantar, através de revisões, relatos de caso e pesquisas, que ajudem a familiarizar o profissional da atenção básica, que faz o primeiro acolhimento do paciente, permitindo o diagnóstico precoce e redução das dificuldades físicas e psicossociais que o paciente enfrenta devido à doença.

\section{REFERÊNCIAS BIBLIOGRÁFICAS}

1. Sociedade Brasileira De Dermatologia (SBD). Consenso Brasileiro de Psoríase 2012: guias de avaliação e tratamento.

pustulosis: a nationwide study using the Japanese national claims database. BMJ Open [Internet]. 2015 Jan [cited Jun 2017]; 5(1):e006450. Available from: https://doi.org/10.1136/bmjopen-2014-006450

4. Silverberg NB. Pediatric psoriasis: an update. Ther Clin Risk Manag. [Internet]. 2009 Nov [cited Jun 2017]; 5:849-856. Available https://www.ncbi.nlm.nih.gov/pmc/articles/PMC2773753/

5. Benjegerdes KE, Hyde K, Kivelevitch D, Mansouri B. Pustular psoriasis: pathophysiology and current treatment perspectives. Psoriasis: Targets and Therapy [Internet]. 2016 Sep [cited Jun 2017]; 2016:6 131-144. Available from: https://doi.org/10.2147/PTT.S98954

6. Razera F, Olm GS, Bonamigo RR. Dermatoses neutrofílicas Parte II. An Bras Dermatol [Internet]. 2011 [cited Jun 2017]; 86(2):195-212. Available from: http://dx.doi.org/10.1590/S0365-05962011000200001

7. Misiak-Galazka M, Wolska H, Rudnicka L. What do we know about palmoplantar pustulosis? J Eur Acad Dermatol Venereol [Internet]. 2017 Jan [cited junho 2017]; 13:13846. Available from: https://doi.org/10.1111/jdv.13846

8. Malathi M, Thappa DM. Pustular Psoriasis in Children - a Review. Nepal J Dermatol Venereol Leprol [Internet]. 2012 [cited junho 2017]; 10(1):1-10 Available from: http://dx.doi.org/10.3126/njdvl.v10i1.6416

9. Stephen K. Palmoplantar pustulosis - an overview. Dermatol Nurs [Internet]. 2017 Mar [cited Jun 2017]; 16(1); 18-2. Available from: http://www.bdng.org.uk

10. Engin B, Aşkın Ö, Tüzün Y. Palmoplantar Psoriasis. Clin Dermatol [Internet]. 2017 Jan [cited Jun 2017]; 35,19-27. Available

from:

http://dx.doi.org/10.1016/j.clindermatol.2016.09.004

11. Raposo I, Torres T. Palmoplantar psoriasis and palmoplantar pustulosis: current treatment and future prospects. Am J Clin Dermatol [Internet]. 2016 Aug [cited Jun 2017]; 17:349-58 Available from: https://doi.org/10.1007/s40257-016-0191-7

12. Ferrara FS, Alvarenga CO, Vidigal MR, Tebcherani AJ, Sanchez APG. Psoríase pustulosa palmoplantar tratada com etanercepte: relato de caso. Rev Med (São Paulo) [Internet]. 2011 Jul [cited Jun 2017]; 90(3):128-32. Available from: http://www.revistas.usp.br/revistadc/article/view/58904/618 $\underline{82}$

13. Robinson A, Van Voorhees AS, Hsu S, Korman NJ, Lebwohl MG, Bebo BF Jr, Kalb RE. Treatment of pustular psoriasis: from the Medical Board of the National Psoriasis Foundation. J Am Acad Dermatol [Internet]. 2012 Aug [cited Jun 2017]; 67(2):279-288. Available from: https://doi.org/10.1016/j.jaad.2011.01.032

14. Farley E, Masrour S, McKey J, Menter A. Palmoplantar psoriasis: a phenotypical and clinical review with introduction of a new quality-of-life assessment tool. J Am Acad Dermatol [Internet]. 2009 Jun [cited Jun 2017]; 60:1024-31. Available from: https://doi.org/10.1016/j.jaad.2008.11.910 